\title{
Beneficial effect of xylo-oligosaccharides and fructo-oligosaccharides in streptozotocin-induced diabetic rats
}

\author{
Duraiswamy Gobinath $^{1}$, Arenahalli N. Madhu ${ }^{1}$, Giribhattanavar Prashant ${ }^{1}$, Krishnapura Srinivasan ${ }^{2}$ \\ and Siddalingaiya G. Prapulla ${ }^{1}$. \\ ${ }^{1}$ Department of Fermentation Technology \& Bioengineering, Central Food Technological Research Institute, CSIR, Mysore \\ 570 020, India \\ ${ }^{2}$ Department of Biochemistry \& Nutrition, Central Food Technological Research Institute, CSIR, Mysore 570 020, India
}

(Received 24 August 2009 - Revised 5 January 2010 - Accepted 11 January 2010 - First published online 26 February 2010)

\begin{abstract}
The beneficial influences of xylo-oligosaccharides (XOS) obtained from alkali-pretreated corncob and fructo-oligosaccharides (FOS) obtained from cane sugar were evaluated in experimental diabetes. These oligosaccharides were supplemented at $10 \%$ (w/w) in the basal diet of streptozotocin-induced diabetic Wistar rats, while the control rats were fed with a basal diet for a period of 6 weeks. Both the oligosaccharides exerted favourable influences in diabetic rats by significantly improving body weight and reducing hyperglycaemia and cholesterol. The characteristic diabetic complications such as severe glucosuria, proteinuria and advanced glycation end products in renal tissue, diabetic nephropathy, and blood creatinine and urea concentrations were notably reduced. Besides, these oligosaccharide supplementations significantly increased the activity of antioxidant enzymes - catalase and glutathione reductase - in the blood of diabetic rats. Supplementation of XOS and FOS resulted in a significant increase in the bifidobacteria and lactobacilli population in the caecum. The present study indicates that XOS and FOS have an ameliorating influence on metabolic abnormalities associated with diabetes, besides conferring an optimal milieu of lactobacilli and bifidobacteria, thus suggesting their potential health benefit in diabetics.
\end{abstract}

Xylo-oligosaccharides: Fructo-oligosaccharides: Diabetes mellitus: Metabolic abnormalities: Nephropathy

Diabetes mellitus is an endocrine disorder that affects over 100 million people worldwide, and is becoming very common with changing lifestyles. Diabetes often leads to disability from the vascular complications of coronary artery disease, cerebrovascular disease, renal failure, limb amputation and blindness, in addition to neurological complications and premature death $^{(1,2)}$. These diabetic complications rank high among the top ten causes of mortality throughout the world. The high fatty acid levels in plasma can lead to the development of atherosclerosis $^{(3)}$ in diabetic patients and a decrease in antioxidant molecules and enzymes, which at the later stages can amplify the diabetic complications ${ }^{(4)}$.

Streptozotocin (STZ)-induced diabetes is a well-recognised diabetic experimental model that causes selective destruction of islet $\beta$-cells, associated with the generation of free radicals ${ }^{(5)}$. Hyperglycaemia is known to contribute to defective $\beta$-cell function in STZ-induced diabetes ${ }^{(6)}$. Dietary intervention with non-digestible fibre is one of the main therapies proposed in the case of diabetic patients ${ }^{(7)}$. A few oligosaccharides and polysaccharides of natural or synthetic origin have gained importance for the treatment of chronic diseases such as IHD ${ }^{(8)}$ and cancer $^{(9)}$. Prebiotics are emerging functional products generally useful for the improvement of the nutritional quality of foods, and are defined as non-digestible food ingredients that beneficially affect the host by selectively stimulating the growth and/or activity of one or a limited number of bacteria in the colon and thus improve host health ${ }^{(10)}$. Prebiotics possess interesting functional and physiological attributes such as low sweetness, non-cariogenicity, low energetic value, and hypolipidaemic and hypocholesterolaemic properties. They are indigestible by human gastrointestinal enzymes and are not degraded by low gastric $\mathrm{pH}$; however, they are fermented in the large bowel and enhance the population of beneficial microbes such as lactobacilli and bifidobacteria ${ }^{(10)}$.

Oligosaccharides such as fructo-oligosaccharides (FOS) are naturally present in wheat, asparagus, garlic, onion, artichoke, soya and milk, though in rather low amounts ${ }^{(11)}$. Xylo-oligosaccharides (XOS) are naturally available in bamboo shoots, which are also produced from xylan, a major component of hemicelluloses ${ }^{(12)}$. FOS and XOS are found to increase the number of beneficial microbes such as bifidobacteria and lactobacilli in the colon and increase the body weight (BW), caecal weight and caecal SCFA ${ }^{(13)}$. FOS and XOS markedly decreased the caecal $\mathrm{pH}$ and serum TAG concentration, and increased total caecal weight and bifidobacteria population $^{(14)}$. Daily intake of FOS $(8 \mathrm{~g} / \mathrm{d})$ for a period of $14 \mathrm{~d}$ reduced blood glucose, cholesterol and LDL in diabetic patients $^{(15)}$. XOS improved growth retardation, hyperphagia,

Abbreviations: AGE, advanced glycation end; BW, body weight; GR, glutathione reductase; FOS, fructo-oligosaccharides; STZ, streptozotocin; XOS, xylo-oligosaccharides.

* Corresponding author: Dr Siddalingaiya G. Prapulla, fax +91 821 2517233, email prapullasg@yahoo.co.in 
polydipsia and elevation of serum glucose, TAG and cholesterol in diabetic rats ${ }^{(16)}$

In the above context, the present investigation evaluated the beneficial effects of two oligosaccharides - XOS and FOS in STZ-induced diabetic rats with respect to glycaemic status, BW, hypercholesterolaemia, activities of antioxidant enzymes and also pathophysiological conditions such as advanced glycation end (AGE) products in renal tissue and kidney pathology. In the absence of any detailed information on these aspects, the present study could provide for a better understanding of the antidiabetic profile of these oligosaccharides.

\section{Experimental methods}

\section{Chemicals}

STZ was procured from ICN Biomedicals Inc. (Asse-Relegem, Belgium). Heparin, glucose oxidase, peroxidase, $o$-dianisidine, bovine serum albumin, Coomassie Brilliant Blue G-250, cysteine hydrochloride, thiosemicarbazide and diacetyl monoxime were purchased from Sigma Chemical Co. (St Louis, MO, USA). Standards of FOS (1-kestose, 1-nystose and 1-fructofuranosyl nystose) were procured from Wako Pure Chemical Industries, Ltd (Osaka, Japan). Casein (refined grade) was procured from Nimesh Corporation (Mumbai, India). Salt mixture was procured from SISCO Research Laboratories Pvt. Ltd (Mumbai, India). All other chemicals and solvents used were of analytical grade.

\section{Production and analysis of oligosaccharides}

FOS (containing 90-93\% (w/w) FOS and designated as FOS-90) consisting of $57 \%$ kestose, $30 \%$ nystose and $5 \%$ fructofuranosyl nystose were prepared by the selective removal of glucose and sucrose (our unpublished results) from FOS-56 (consisting of $56 \%$ (w/w) FOS), which were derived previously from cane sugar using fungal fructosyl transferase $^{(17)}$. XOS (consisting of $90-92 \%$ (w/w) XOS) were prepared through a slight modification of the procedure used by Aachary \& Prapulla ${ }^{(18)}$ under optimised conditions using commercial xylanase (Bioxyl P-40, Biocon Ltd, Bangalore, India). These two oligosaccharides were analysed by HPLC (LC-6A, Shimadzu, Kyoto, Japan) with a refractive index detector using a polar-bonded phase column $\left(\right.$ Exsil $\mathrm{NH}_{2}$, $4.6 \mathrm{~mm} \times 25 \mathrm{~cm}, 5 \mu \mathrm{m})$ at an ambient temperature using acetonitrile-water $(75: 25)$ as a mobile phase at a flow rate of $1.0 \mathrm{ml} / \mathrm{min}$. FOS were identified and quantified by comparing with standards of kestose, nystose and fructofuranosyl nystose ${ }^{(17)}$, while XOS were estimated according to Jeong et al. ${ }^{(19)}$ and Aachary \& Prapulla ${ }^{(18)}$. The refractive index detector used for the analysis of FOS and XOS has been standardised in our laboratory, and it was found to be satisfactory ${ }^{(17,18)}$.

\section{Animals and dietary treatment}

The present animal study was carried out by taking all appropriate precautions and by strictly following the guidelines with regard to the use of animals for experimental purpose after due approval from the Institutional Animal Ethics Committee (CFTRI, Mysore, India). Influence of prebiotics on diabetic rats was examined using male Wistar rats (150-160g) procured from the animal production facility of this institute. Rats were divided into the following groups: (1) control group fed with the basal diet; (2) control group fed with the basal diet containing XOS (10\%); (3) control group fed with the basal diet containing FOS (10\%); (4) control group fed with the basal diet containing XOS (5\%) + FOS (5\%); (5) diabetic control group fed with the basal diet; (6) diabetic group fed with basal diet containing XOS (10\%); (7) diabetic group fed with the basal diet containing FOS (10\%); (8) diabetic group fed with the basal diet containing XOS $(5 \%)+$ FOS $(5 \%)$. The basal diet consisted $(\%)$ of maize starch, 54; casein, 21; refined peanut oil, 10; powdered cane sugar, 10; Bernhardt-Tommarelli salt mixture, 4; and National Research Council (NRC) vitamin mixture, 1. The oligosaccharide diets contained either XOS $(10 \%)$ or FOS $(10 \%)$, or a combination of XOS $(5 \%)$ and FOS $(5 \%)$ at the $10 \%$ level by replacing an equivalent amount of maize starch in the basal diet (w/w).

\section{Induction of diabetes}

Diabetes was induced by a single administration of STZ (intraperitonially $40 \mathrm{mg} / \mathrm{kg} \mathrm{BW}$ in $1 \mathrm{ml}$ of $0 \cdot 1 \mathrm{M}$-citrate buffer, $\mathrm{pH} 4.5)$ to overnight fasted rats. A parallel set of control rats (non-diabetic) were injected with citrate buffer only. Glucose $(5 \%)$ was given for $48 \mathrm{~h}$ following the intra-peritonial injection of STZ to prevent initial drug-induced hypoglycaemic mortality. Blood was drawn from the retro-orbital plexus 1 week after STZ administration, and it was used for the determination of fasting blood glucose. Animals having at least two and half times the normal fasting blood glucose were considered as hyperglycaemic.

The groups of rats were allowed to access the respective food and water ad libitum for 6 weeks. BW was recorded weekly. Twenty-four-hour urine samples were collected at weekly intervals from individual rats housed in metabolic cages, filtered and stored at $-20^{\circ} \mathrm{C}$ for further analysis of urinary protein and glucose excretion. Blood glucose was monitored biweekly, while urinary excretion of glucose and protein was monitored weekly.

At the end of the experiment, rats were killed, and blood was collected in heparinised tubes (20 $\mathrm{U}$ heparin/ml blood) and centrifuged at $4^{\circ} \mathrm{C}$ at $4000 \mathrm{rpm}$ for $10 \mathrm{~min}$ (Remi centrifuge, Mumbai, India). The separated plasma was stored at $-20^{\circ} \mathrm{C}$ until processed. The caecum samples were collected and weighed wet. An aliquot from caecal content was used for microbial analysis, and the remainder was stored at $-20^{\circ} \mathrm{C}$ for further analysis. The kidneys were excised, weighed, homogenised in phosphate buffer using a Potter-Elvehjem homogeniser, and were then centrifuged at $8000 \mathrm{rpm}$ at $4^{\circ} \mathrm{C}$ for $20 \mathrm{~min}$. The supernatant was stored at $-20^{\circ} \mathrm{C}$ for further analysis.

\section{Caecal characteristics}

After dissection, the rat caecum was immediately removed, and total net weight was recorded, and then it was stored at a refrigerated temperature for further analysis. Bifidobacteria and lactobacilli were enumerated from a known quantity of suitably diluted caecal matter using spread plate method in selective Bifidobacterium iodoacetate agar (anaerobically, 
Table 1. Effect of prebiotics on growth in diabetic rats (Mean values and standard deviations of eight rats)

\begin{tabular}{|c|c|c|c|c|c|c|}
\hline \multirow[b]{3}{*}{ Animal group } & \multicolumn{4}{|c|}{ Body weight (g) } & & \\
\hline & \multicolumn{2}{|c|}{ Initial (0d) } & \multicolumn{2}{|c|}{ Final (6th week) } & \multicolumn{2}{|c|}{ Weight gain (g) } \\
\hline & Mean & SD & Mean & SD & Mean & SD \\
\hline Diabetic control & $161 \cdot 1^{a}$ & $2 \cdot 9$ & $123 \cdot 8^{\mathrm{a}}$ & $7 \cdot 2$ & $-37 \cdot 3^{a}$ & $6 \cdot 1$ \\
\hline Diabetic XOS & $162 \cdot 9^{a}$ & 6.9 & $142 \cdot 5^{\mathrm{b}}$ & $6 \cdot 1$ & $-19.9^{b}$ & $10 \cdot 2$ \\
\hline Diabetic FOS & $162 \cdot 4^{\mathrm{a}}$ & $4 \cdot 1$ & $141 \cdot 8^{\mathrm{b}}$ & $8 \cdot 3$ & $-20 \cdot 6^{\mathrm{b}}$ & 6.9 \\
\hline Diabetic XOS + FOS & $162 \cdot 1^{\mathrm{a}}$ & 6.6 & $142 \cdot 6^{\mathrm{b}}$ & $8 \cdot 8$ & $-19 \cdot 5^{b}$ & 9.9 \\
\hline Normal control & $168 \cdot 8^{\mathrm{a}}$ & $8 \cdot 3$ & $257.8^{\mathrm{C}}$ & $4 \cdot 2$ & $89.8^{\mathrm{c}}$ & 8.2 \\
\hline Normal XOS & $165 \cdot 8^{\mathrm{a}}$ & 9.5 & $265 \cdot 7^{c}$ & $5 \cdot 3$ & $99.9^{c}$ & 5.4 \\
\hline Normal FOS & $164 \cdot 0^{\mathrm{a}}$ & 8.1 & $262 \cdot 2^{c}$ & 3.2 & $98 \cdot 2^{c}$ & 4.9 \\
\hline Normal XOS + FOS & $165 \cdot 7^{\mathrm{a}}$ & $7 \cdot 4$ & $263 \cdot 5^{\mathrm{c}}$ & 3.8 & $97 \cdot 8^{\mathrm{C}}$ & 6.7 \\
\hline
\end{tabular}

XOS, xylo-oligosaccharides; FOS, fructo-oligosaccharides.

a,b,c Mean values within a column with unlike superscript letters were significantly different $(P \leq 0.05)$.

$37^{\circ} \mathrm{C}$ for $48 \mathrm{~h}$ ) and Lactobacillus MRS (de Mann Rogosa) agar (aerobically, $37^{\circ} \mathrm{C}$ for $24 \mathrm{~h}$ ), respectively. Microbial counts were expressed as log colony-forming units/g wet sample. Aseptic conditions were maintained throughout the microbial enumeration. The $\mathrm{pH}$ of the caecal matter was also recorded (Analab Scientific Instrument Pvt. Ltd, Vadodara, India).

\section{Analytical parameters}

Fasting blood glucose and glucose excretion in urine were determined by the glucose oxidase and peroxidase method ${ }^{(20)}$. Total plasma protein and protein excretion in urine were analysed by the Bradford method ${ }^{(21)}$. The method described by Folin \& $\mathrm{Wu}^{(22)}$ was used to estimate plasma and urinary creatinine. Plasma urea was estimated as described by Levine $^{(23)}$, and urinary urea was measured as described by Wyebenga et al. ${ }^{(24)}$. Formation of AGE products in renal tissue was determined as reported by Monnier \& Cerami ${ }^{(25)}$. Plasma total cholesterol was determined as described by Rudel $\&$ Morris $^{(26)}$. Activity of catalase was assayed by the method of Takahara et al. ${ }^{(27)}$, and glutathione reductase (GR) activity was assayed using a standard procedure ${ }^{(28)}$. All the experiments were carried out in triplicate.

\section{Histological studies}

Light microscopic observations were made with haematoxylin-eosin-stained thin sections of kidney previously fixed in $10 \%$ formalin and embedded in paraffin.

\section{Statistical analysis}

Values were expressed as means and standard deviations of eight rats. Statistical analysis was carried out using Origin 6.1 statistical software (Originlab Corporation, Northampton, MA, USA). Results were analysed and the significance level was calculated using the Tukey-Kramer multiple comparison test, and results are considered significant at $P<0 \cdot 05$.

\section{Results}

Effect of xylo-oligosaccharides and fructo-oligosaccharides on body weight and mortality

The influence of prebiotic supplementation on the BW of diabetic rats and normal rats is presented in Table 1. Gain in BW was markedly suppressed in diabetic rats, whereas the diabetic rats fed with XOS and FOS diets showed a significant improvement in the BW during the feeding

Table 2. Effect of prebiotics on total caecum weight, $\mathrm{pH}$, bifidobacteria and lactobacilli population of the caecum contents (Mean values and standard deviations of eight rats)

\begin{tabular}{|c|c|c|c|c|c|c|c|c|}
\hline \multirow[b]{2}{*}{ Animal group } & \multicolumn{2}{|c|}{$\begin{array}{l}\text { Total caecum weight } \\
\text { (g) }\end{array}$} & \multicolumn{2}{|c|}{$\mathrm{pH}$} & \multicolumn{2}{|c|}{ Bifidobacteria* } & \multicolumn{2}{|c|}{ Lactobacilli* } \\
\hline & Mean & SD & Mean & SD & Mean & SD & Mean & SD \\
\hline Diabetic control & $2.05^{a}$ & 0.12 & $6 \cdot 73^{d}$ & 0.16 & $8 \cdot 16^{a}$ & $0 \cdot 11$ & $7 \cdot 04^{a}$ & 0.12 \\
\hline Diabetic XOS & $2 \cdot 55^{b}$ & 0.11 & $6 \cdot 49^{a, b}$ & $0 \cdot 15$ & $9 \cdot 11^{d}$ & 0.14 & $7 \cdot 47^{\mathrm{b}}$ & 0.16 \\
\hline Diabetic FOS & $2 \cdot 41^{b}$ & 0.23 & $6 \cdot 55^{\mathrm{a}, \mathrm{b}}$ & 0.19 & $8 \cdot 67^{b}$ & $0 \cdot 15$ & $7 \cdot 82^{c}$ & 0.24 \\
\hline Diabetic XOS + FOS & $2 \cdot 43^{b}$ & 0.16 & $6 \cdot 52^{\mathrm{a}, \mathrm{b}}$ & 0.21 & $9 \cdot 03^{c, d}$ & 0.09 & $7 \cdot 62^{\mathrm{b}, \mathrm{c}}$ & 0.14 \\
\hline Normal control & $2.92^{b}$ & 0.41 & $6 \cdot 65^{\mathrm{b}, \mathrm{c}}$ & 0.11 & $8 \cdot 89^{b, c}$ & 0.21 & $7 \cdot 45^{\mathrm{b}}$ & 0.16 \\
\hline Normal XOS & $4 \cdot 05^{d}$ & 0.21 & $6 \cdot 35^{\mathrm{a}}$ & 0.08 & $10 \cdot 2^{f}$ & 0.12 & $7 \cdot 81^{c}$ & 0.23 \\
\hline Normal FOS & $3 \cdot 61^{c}$ & 0.28 & $6 \cdot 42^{\mathrm{a}, \mathrm{c}}$ & 0.14 & $9 \cdot 87^{\mathrm{e}}$ & 0.22 & $8 \cdot 21^{d}$ & 0.16 \\
\hline Normal XOS + FOS & $3.93^{c, d}$ & 0.33 & $6 \cdot 38^{a}$ & $0 \cdot 10$ & $10 \cdot 1^{f}$ & $0 \cdot 10$ & $8 \cdot 04^{d}$ & 0.11 \\
\hline
\end{tabular}

XOS, xylo-oligosaccharides; FOS, fructo-oligosaccharides.

$\mathrm{a}, \mathrm{b}, \mathrm{c}, \mathrm{d}, \mathrm{e}, \mathrm{f}$ Mean values within a column with unlike superscript letters were significantly different $(P \leq 0.05)$.

${ }^{*} \log _{10}$ colony-forming units/g caecal wet content. 
period in comparison with those fed with the basal diet. The BW of the control rats fed with oligosaccharide diets was not significantly increased compared with those fed with the basal diet. High mortality was generally observed in diabetic rats (as much as 50\%) fed with the basal diet, but oligosaccharide supplementation reduced the mortality by up to 8 , 14 and $21 \%$ in the case of FOS (10\%), XOS (10\%) and combination of XOS $(5 \%)$ and FOS $(5 \%)$ in diabetic rats, respectively (data not presented). Interestingly, the mortality was less in the case of FOS-fed diabetic rats compared with other diabetic groups.

\section{Effect of prebiotics on caecal characteristics}

Data on caecal wet weight, $\mathrm{pH}$, bifidobacteria and lactobacilli count are presented in Table 2. Total wet weight of the caecum was significantly $(P \leq 0.05)$ increased in diabetic and non-diabetic rats consuming XOS and FOS than in the rats fed with the basal diet. In addition, rats fed with oligosaccharides showed a significant decrease in $\mathrm{pH}$ from 6.73 (SD 1.6) up to 6.35 (SD 0.08) and an increase in the bifidobacteria and lactobacilli count in the caecum compared with rats fed with the basal diet. Rats fed with the XOS-containing diet, either $10 \%$ or $5 \%$, showed a significant increase in bifidobacteria compared with rats fed with FOS $(10 \%)$. The rats fed with the FOS-containing diet, either 10 or $5 \%$, showed a significant concentration of lactobacilli compared to rats fed with XOS. Thus, the result explains the prebiotic efficacy of XOS and FOS.

\section{Effect of xylo-oligosaccharides and fructo-oligosaccharides} on fasting glucose, cholesterol, creatinine and urea in plasma

Hyperglycaemia was ameliorated throughout the experimental period in the case of diabetic rats fed with the XOS or FOS diet. Treatment of diabetic rats with prebiotics at the $10 \%$ dietary level for a period of 6 weeks brought down hyperglycaemia significantly $(P \leq 0.05$; Fig. 1$)$, and a similar

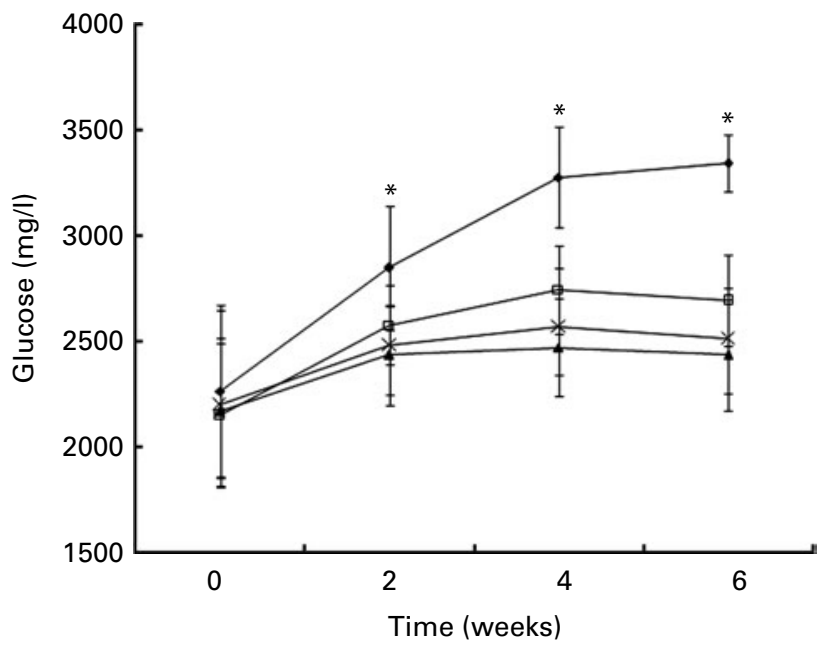

Fig. 1. Fasting blood glucose in diabetic rats fed with prebiotics. Diabetic control (- -); diabetic xylo-oligosaccharides (XOS, - $\square-$ ); diabetic fructo-oligosaccharides (FOS, $-\boldsymbol{\Delta}-$ ) and diabetic XOS + FOS $(-\times-$ ). * Mean values are significantly different from those of diabetic rats fed with prebiotic groups $(P \leq 0.05)$.

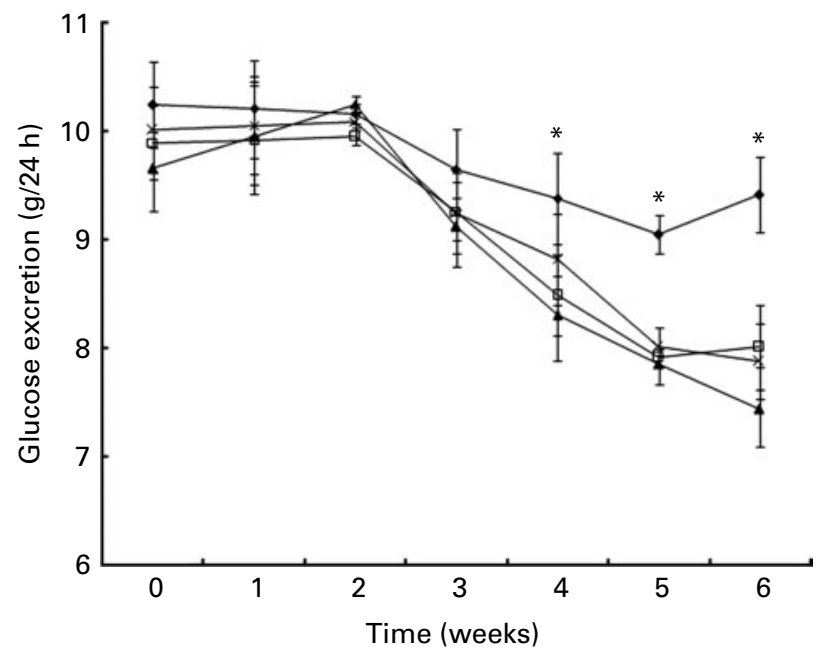

Fig. 2. Urinary glucose excretion in diabetic rats fed with prebiotics. Diabetic control $(-\downarrow)$ ); diabetic xylo-oligosaccharides (XOS, $-\square-$ ); diabetic fructooligosaccharides (FOS, $-\mathbf{\Delta}-$ ) and diabetic XOS + FOS $(-\times-)$. * Mean values are significantly different from those of diabetic rats fed with prebiotic groups $(P \leq 0.05)$.

trend was observed in the urinary glucose excretion pattern in oligosaccharide-fed diabetic rats (Fig. 2). In addition, oligosaccharide diets significantly $(P \leq 0.05)$ reduced the plasma cholesterol, creatinine and urea concentration in diabetic rats in comparison with diabetic rats fed with basal diet. Effects on plasma cholesterol, creatinine and urea are presented in Table 3.

Effect of xylo-oligosaccharides and fructo-oligosaccharides on plasma protein, kidney weight and advanced glycation end products

Table 3 shows the effect of XOS and FOS on plasma protein and kidney weight in rats. Diabetic rats fed with the basal diet showed a high degree of plasma protein depletion (59.0 (SD 2.9) $\mathrm{mg} / \mathrm{l}$ ) compared with control rats (65.6 (SD 1.1) $\mathrm{mg} / \mathrm{l})$. In diabetic rats fed with oligosaccharides, the plasma protein destruction was reduced up to $3-4 \%$ compared with diabetic control rats. The urinary protein excretion pattern substantiates the above finding (Fig. 3). Countering of proteinuria by dietary oligosaccharides in diabetic rats was progressive with the duration of the diet regimen, and it was maximum in the FOS group. As is typical in diabetes, the increase in the kidney weight in diabetic rats was markedly high compared with the control rats. A reduction in kidney weight was observed in the case of diabetic rats fed with oligosaccharides compared with diabetic control rats. The increased kidney weight corresponds to the presence of diabetic nephropathy such as heavy AGE product formation and subsequent damage in renal tissues. XOS and FOS diets also decreased the level of AGE products in renal tissue of diabetic rats compared with those fed with the basal diet. The relative intensity of 15.3 for diabetic controls corresponds to an increased number of AGE products, which was found to be significantly reduced to a relative intensity of $12.83,13.20$ and 13.14 at $440 \mathrm{~nm}$ in the diabetic rats fed with XOS, FOS and a combination of XOS + FOS, respectively. The control rats fed with prebiotics showed a relative intensity in the range of $10 \cdot 8-10 \cdot 9$, which 
Table 3. Effect of prebiotics on protein, creatinine, urea, cholesterol in plasma and kidney weight (Mean values and standard deviations of eight rats)

\begin{tabular}{|c|c|c|c|c|c|c|c|c|c|c|}
\hline \multirow[b]{3}{*}{ Animal group } & \multicolumn{8}{|c|}{ Plasma } & & \\
\hline & \multicolumn{2}{|c|}{$\begin{array}{l}\text { Protein } \\
(\mathrm{g} / \mathrm{l})\end{array}$} & \multicolumn{2}{|c|}{$\begin{array}{l}\text { Creatinine } \\
(\mathrm{mg} / \mathrm{l})\end{array}$} & \multicolumn{2}{|c|}{$\begin{array}{l}\text { Urea } \\
(\mathrm{mg} / \mathrm{l})\end{array}$} & \multicolumn{2}{|c|}{$\begin{array}{l}\text { Cholesterol } \\
(\mathrm{mg} / \mathrm{l})\end{array}$} & \multicolumn{2}{|c|}{$\begin{array}{l}\text { Kidney weight } \\
\text { (g/100 g BW) }\end{array}$} \\
\hline & Mean & SD & Mean & SD & Mean & SD & Mean & SD & Mean & SD \\
\hline Diabetic control & $59 \cdot 0^{\mathrm{a}}$ & $1 \cdot 7$ & $25 \cdot 7^{\mathrm{c}}$ & $2 \cdot 7$ & $450^{\mathrm{C}}$ & $28 \cdot 3$ & $2295^{\mathrm{c}}$ & 175 & $0.981^{a}$ & 0.158 \\
\hline Diabetic XOS & $62 \cdot 9^{\mathrm{b}}$ & 1.5 & $20 \cdot 1^{\mathrm{b}}$ & $1 \cdot 1$ & $422^{\mathrm{b}}$ & $24 \cdot 1$ & $2146^{b}$ & 101 & $0.843^{b}$ & 0.128 \\
\hline Diabetic FOS & $63 \cdot 3^{b}$ & $1 \cdot 1$ & $19 \cdot 4^{b}$ & $2 \cdot 8$ & $418^{\mathrm{b}}$ & 23.9 & $2087^{b}$ & 166 & $0.837^{\mathrm{b}}$ & 0.093 \\
\hline Diabetic XOS + FOS & $62 \cdot 9^{\mathrm{b}}$ & 1.7 & $19 \cdot 7^{\mathrm{b}}$ & $2 \cdot 1$ & $418^{\mathrm{b}}$ & $16 \cdot 7$ & $2111^{b}$ & 171 & $0.839^{b}$ & 0.206 \\
\hline Normal control & $65 \cdot 6^{c}$ & 1.1 & $10 \cdot 4^{a}$ & $2 \cdot 2$ & $391^{a}$ & $6 \cdot 6$ & $1817^{a}$ & 118 & $0.379^{c}$ & 0.048 \\
\hline Normal XOS & $66 \cdot 1^{\mathrm{c}}$ & 1.5 & $10 \cdot 3^{a}$ & $1 \cdot 1$ & $388^{a}$ & 2.5 & $1771^{\mathrm{a}}$ & 69 & $0.361^{c}$ & 0.023 \\
\hline Normal FOS & $66 \cdot 3^{c}$ & 1.2 & $10 \cdot 2^{\mathrm{a}}$ & $2 \cdot 0$ & $385^{a}$ & $12 \cdot 8$ & $1744^{a}$ & 104 & $0.363^{c}$ & 0.017 \\
\hline Normal XOS + FOS & $66 \cdot 0^{c}$ & 1.6 & $10 \cdot 2^{\mathrm{a}}$ & $2 \cdot 1$ & $384^{\mathrm{a}}$ & 8.9 & $1754^{\mathrm{a}}$ & 76 & $0.364^{c}$ & 0.026 \\
\hline
\end{tabular}

BW, body weight; XOS, xylo-oligosaccharides; FOS, fructo-oligosaccharides.

a,b,c Mean values within a column with unlike superscript letters were significantly different $(P \leq 0.05)$.

was comparable to that shown by normal control rats fed with the basal diet.

\section{Histopathology of kidney sections}

Histological examination of the kidney sections revealed pronounced glomerulosclerosis and tubular lesions and cellular infiltration in all diabetic rats maintained on the basal diet. The diabetic rats fed with XOS and FOS showed a decreased degree of renal pathology compared with diabetic rats fed with the basal diet (Fig. 4).

\section{Effect of xylo-oligosaccharides and fructo-oligosaccharides} on the activities of plasma catalase and glutathione reductase

Results showed that the activity of antioxidant enzymes, with catalase and GR, was lowered in diabetic rats compared with normal control rats, but diabetic rats fed with oligosaccharides showed improved $(P \leq 0.05)$ activities of catalase and GR compared with diabetic control rats fed with the basal diet (Table 4). Such an increase, however, was not observed in normal rats fed with XOS or FOS.

\section{Discussion}

In the present study, consumption of oligosaccharides FOS and XOS showed desirable effects in STZ-induced diabetic rats. Oligosaccharide diets markedly improved the BW in diabetic rats, reduced the mortality and significantly increased the bifidobacteria and lactobacilli population in the caecum. The tested oligosaccharides at $10 \%$ dietary concentration beneficially countered fasting hyperglycaemia associated with diabetes, improved plasma albumin and significantly attenuated the rise in plasma concentrations of cholesterol, creatinine and urea. In addition, diabetes-induced reduction in the activity of antioxidant enzymes catalase and GR in plasma was ameliorated. Attenuation of diabetic nephromegaly by dietary oligosaccharides was accompanied by a reduction in AGE products in the renal tissue. Dietary levels of XOS and FOS $(10 \%)$ used in the present study conform to the levels used in our previous report on the beneficial effect of FOS $^{(29)}$. Health beneficial hypocholesterolaemic and antidiabetic influences of various dietary fibres included up to $10 \%$ in the diet in animal studies have been reported by various authors without any adverse effects. Thus, we used the $10 \%$ level of oligosaccharides either individually or in combination.

While dietary XOS and FOS resulted in a similar influence on various parameters studied, a striking difference between them was their effect on microbial flora. While FOS stimulated the growth of lactobacilli, XOS stimulated the growth of bifidobacteria. Several in vitro and in vivo studies have demonstrated that diets containing oligosaccharides such as FOS and XOS selectively increase the bifidobacteria and lactobacilli population, and decrease the $\mathrm{pH}$ of caecal content ${ }^{(10,13,14)}$. In the present study, their dietary supplementation at the $10 \%$ level significantly increased the caecum total weight, bifidobacteria and lactobacilli population, and decreased the $\mathrm{pH}$ of the caecal content. The reduction in the $\mathrm{pH}$ of caecal content after the supplementation of prebiotics could be attributed to an increase in the SCFA concentration

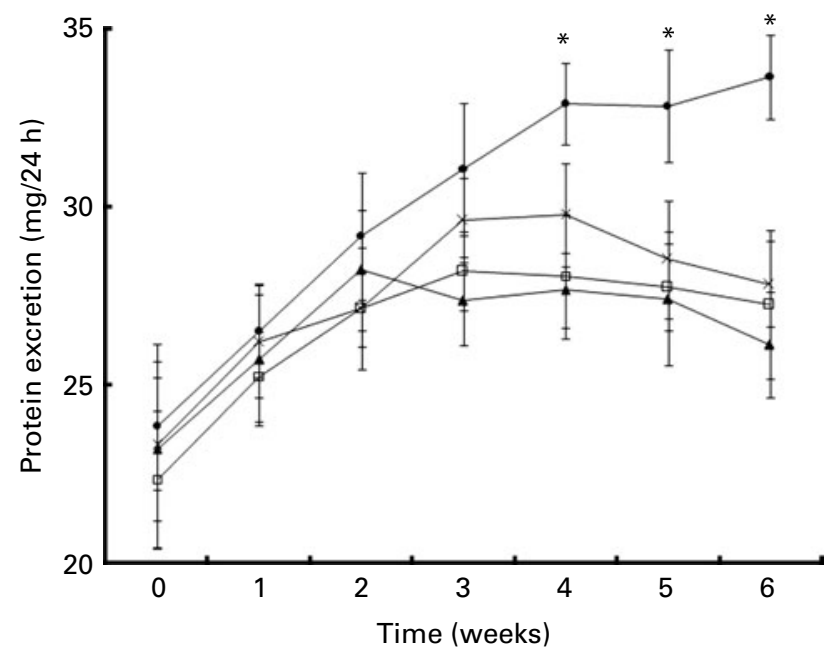

Fig. 3. Urinary protein excretion in diabetic rats fed with prebiotics. Diabetic control $(-\bullet-)$; diabetic xylo-oligosaccharides (XOS, $-\square-$ ); diabetic fructooligosaccharides (FOS, $-\mathbf{\Delta -}$ ) and diabetic-XOS + FOS $(-\times-)$. * Mean values are significantly different from those of diabetic rats fed with prebiotic groups $(P \leq 0.05)$. 
(a)

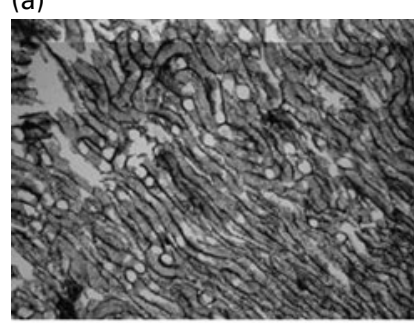

(c)

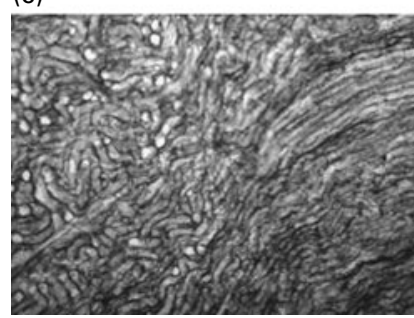

(b)

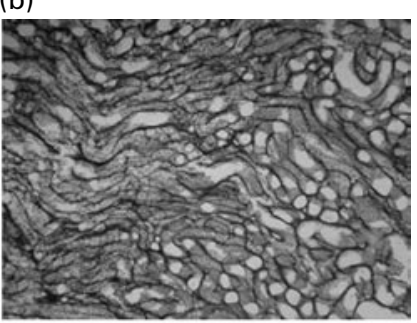

(d)

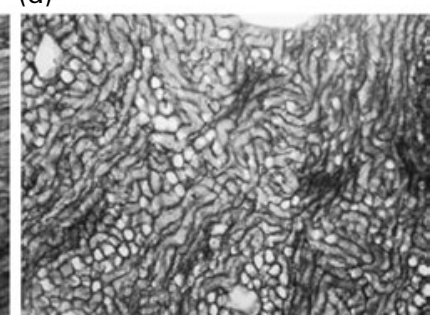

Fig. 4. Kidney sections of diabetic rats maintained on prebiotics (stained with haematoxylin-eosin; $\times 100$ magnification). (a) Normal control (normal renal morphology); (b) diabetic control (characterised by increased glomerulosclerosis and tubular lesions); (c) normal xylo-oligosaccharides (XOS) + fructo-oligosaccharides (FOS; normal renal morphology); (d) diabetic XOS + FOS (showing reduced renal pathology).

by fermentation of bifidobacteria and lactobacilli. Among the two oligosaccharides, XOS was found to be more bifidogenic, which is in agreement with earlier reports of XOS $(1 \mathrm{~g} / \mathrm{d})$ producing a selective increase in bifidobacteria ${ }^{(30-32)}$. The growth of lactobacilli was enhanced by FOS as observed previously by Campbell et al. ${ }^{(13)}$. These beneficial microflora which can utilise XOS and FOS produce SCFA such as lactate, acetate, propionate and butyrate, which in turn influence the glucose and lipid metabolism ${ }^{(33,34)}$.

The present study has shown that with the administration of XOS or FOS for a period of 6 weeks, the plasma glucose level was significantly decreased in the diabetic rats. The reduction in high glucose level was observed in diabetic rats from the second week of prebiotic supplementation, and the reduction was found to be maximum in FOS-fed diabetic rats at 6 weeks. It has been reported earlier that the intake of FOS

Table 4. Effect of prebiotics on plasma catalase and glutathione reductase activities in diabetic rats*

(Mean values and standard deviations of eight rats)

\begin{tabular}{|c|c|c|c|c|}
\hline \multirow[b]{2}{*}{ Groups } & \multicolumn{2}{|c|}{ Catalase } & \multicolumn{2}{|c|}{$\begin{array}{l}\text { Glutathione } \\
\text { reductase }\end{array}$} \\
\hline & Mean & SD & Mean & SD \\
\hline Diabetic control & $3287^{a}$ & 109 & $2524^{a}$ & 13 \\
\hline Diabetic XOS & $3498^{b}$ & 88 & $2606^{b}$ & 29 \\
\hline Diabetic FOS & $3549^{b}$ & 75 & $2646^{b}$ & 31 \\
\hline Diabetic XOS + FOS & $3508^{b}$ & 98 & $2621^{b}$ & 22 \\
\hline Normal control & $4221^{\mathrm{c}}$ & 91 & $3028^{c}$ & 97 \\
\hline Normal XOS & $4319^{c}$ & 79 & $3109^{c}$ & 26 \\
\hline Normal FOS & $4373^{c}$ & 81 & $3097^{c}$ & 31 \\
\hline Normal XOS + FOS & $4331^{c}$ & 106 & $3152^{c}$ & 36 \\
\hline
\end{tabular}

XOS, xylo-oligosaccharides; FOS, fructo-oligosaccharides.

a,b,c Mean values within a column with unlike superscript letters were significantly different $(P \leq 0.05)$.

${ }^{*}$ All values are expressed as units/l.

(syrup containing $56 \% \mathrm{FOS}$ ) at 10 and $5 \%$ in the diet for a period of 6 weeks did not affect the blood glucose level in diabetic rats ${ }^{(32)}$. The positive effect of FOS on the severity of hyperglycaemia in diabetic rats observed in the present study is in contrast to an earlier report ${ }^{(29)}$, possibly because of the higher concentration of FOS (syrup containing $90 \%$ FOS) in the present study compared with FOS (syrup containing $56 \%$ ) used earlier. Intake of FOS $(8 \mathrm{~g} / \mathrm{d})$ for a period of $14 \mathrm{~d}$ has been reported to decrease fasting blood glucose level in diabetic subjects ${ }^{(15)}$. Dietary supplementation with XOS $(4 \mathrm{~g} / \mathrm{d})$ improved blood sugar and lipids in type 2 diabetes $^{(35)}$. These two reports and the present study thus suggest that FOS- or XOS-containing diets are beneficial to diabetic subjects in alleviating the severity of hyperglycaemia.

In the present study, the dietary oligosaccharides significantly reduced the plasma cholesterol level in diabetic rats. It is suggested that the oligosaccharides could reduce blood cholesterol by reducing the cholesterol absorption and increasing the excretion of bile acid and cholesterol in faeces ${ }^{(36)}$ or by decreasing the enzymes involved in fatty acid synthesis ${ }^{(37)}$. Several studies have shown that intake of FOS reduces TAG and cholesterol associated with LDL and VLDL ${ }^{(38)}$. Supplementation of XOS $(10 \%)$ in diet for a period of 5 weeks significantly reduced cholesterol and TAG in diabetic rats ${ }^{(16)}$, and also XOS $(4 \mathrm{~g} / \mathrm{d})$ reduced the total cholesterol and LDL-cholesterol values in type 2 diabetes ${ }^{(35)}$. It has been reported that plasma cholesterol and TAG might be reduced by an increase in the amounts of SCFA (propionic acid) in the large intestine during fermentation of non-digestible carbohydrates ${ }^{(39)}$, and it has been demonstrated that propionic acid supplementation decreased the fasting serum glucose and insulin sensitivity and influenced lipid metabolism in healthy volunteers ${ }^{(33)}$. The decrease in plasma cholesterol in diabetic rats fed with XOS or FOS could be due to the inhibition of cholesterol synthesis by an increase in propionate or modifications in the bile acid metabolism during oligosaccharide supplementation, and hence it can be used as an adjunct against hypercholesterolaemia.

Plasma creatinine level was higher in diabetic rats compared with normal animals, which is an indication of increased muscle wasting during this metabolic disorder. Dietary oligosaccharides lowered the blood creatinine concentration in diabetic rats. This indicates that these oligosaccharide diets counter muscle wasting normally associated with diabetes mellitus, and this is in concurrence with the improvement in BW observed in all the experimental diet-fed diabetic rats. Urea is another end product of the metabolic process and high blood urea levels indicate the impaired renal function, and oligosaccharide supplementation lowered the plasma urea concentration compared with that of diabetic control rats. A study demonstrated that the addition of oligosaccharides and gum arabic to the diet decreased blood urea by $20-30 \%$ and renal nitrogen excretion relative to the control ${ }^{(40)}$.

In the present study, oligosaccharide supplementations were found to considerably protect this loss of protein in blood as indicated by decreased proteinuria in diabetic rats towards the end of the experimental period. These results are in agreement with the earlier study, wherein an intake of FOS $(56 \%)$ at 10 and $5 \%$ dietary levels for a period of 6 weeks was found to protect diabetic rats from the urinary loss of albumin compared with diabetic rats fed with the basal diet ${ }^{(29)}$. 
An abnormally elevated blood glucose level causes oxidative stress and leads to the formation of AGE products ${ }^{(41)}$. Dietary oligosaccharides reduced the formation of AGE products in the renal tissue of diabetic rats compared with those fed with the basal diet, suggesting that it would inhibit oxidative damage caused by the protein glycation reaction under diabetic conditions. These results showed that the administration of prebiotics at the $10 \%$ dietary level might effectively alleviate the pathogenesis of diabetic complications caused by impaired glucose metabolism and the glycosylation of tissue proteins, eventually resulting in an alleviation of the diabetic pathological conditions. The increased weight of kidney in diabetic rats was also countered in oligosaccharide-fed diabetic animals. Administration of these test materials for 6 weeks reduced protein depletion, nephromegaly and glycation of renal tissue proteins. Light microscopy of kidney sections revealed nephromegaly and damaged glomeruli and basement membrane in diabetic rats. The magnitude of these changes was less in FOS- or XOS-fed diabetic rats. Presumably, this beneficial ameliorating influence of dietary oligosaccharides on diabetic nephropathy is attributable to their lowering effects on blood cholesterol levels ${ }^{(41)}$.

In the present study, the activity of catalase in the blood of diabetic rats fed with FOS or XOS was significantly $(P \leq 0 \cdot 05)$ higher compared with diabetic rats fed with the basal diet. In contrast, supplementation of XOS $(4 \mathrm{~g} / \mathrm{d})$ has been reported to reduce the activity of catalase in erythrocytes but not the activity of superoxide dismutase and glutathione peroxidase in type 2 diabetes $^{(42)}$. GR is another important oxidative defence enzyme, which converts the GSSG to GSH, an antioxidant molecule. Reduction of GSSG to GSH was found to be decreased in diabetic rats compared with diabetic rats fed with prebiotic diets. Prebiotic-mediated protection of plasma protein could be implied in improved activity of catalase and GR. The increase in GR activity in blood in turn neutralises superoxide anions and counteracts oxidative stress in diabetes.

\section{Conclusions}

The present investigation indicates that the supplementation of oligosaccharides (XOS and FOS) at the $10 \%$ dietary level confers beneficial effects in STZ-induced diabetic rats with respect to plasma glucose, cholesterol and several other metabolic parameters. The reduced hyperglycaemia and hypercholesterolaemia by oligosaccharides may have in turn contributed to a decrease in the AGE products in the renal tissue and lowered nephromegaly in diabetic rats. The present study indicates that XOS and FOS at the $10 \%$ dietary level can be used as an adjunct to dietary therapy to derive antidiabetic benefits, and to delay secondary complications. A detailed study on the mechanisms of these beneficial effects exerted by these oligosaccharides would be challenging and merits further investigation.

\section{Acknowledgements}

The authors thank Life-science Research Board, DRDO, New Delhi, for funding the project. The animal study was designed by S. G. P. and K. S. D. G., A. N. M. and G. P. performed the animal study, analytical work and statistical analysis of data. D. G. drafted the manuscript; K. S. and S. G. P. reviewed and interpreted the experimental data and refined the write-up of the present manuscript. The authors thank the Director of CFTRI for supporting the work. There are no conflicts of interest whatsoever among the authors.

\section{References}

1. Goldstein DA \& Massry SG (1978) Diabetic nephropathy: clinical course and effect of hemodialysis. Nephron 20, 286-296.

2. Weidmann P, Boehlen LM \& de Courten M (1993) Pathogenesis and treatment of hypertension associated with diabetes mellitus. Am Heart J 125, 1498-1513.

3. Steinberg HO, Paradisi G, Hook G, et al. (2000) Free fatty acid elevation impairs insulin-mediated vasodilation and nitric oxide production. Diabetes 49, 1231-1238.

4. West I (2000) Radicals and oxidative stress in diabetes. Diabetes Med 17, 171-180.

5. Thomas G \& Ramwell PW (1989) Streptozotocin: a nitric oxide-carrying molecule and its effects on vasodilation. Eur $J$ Pharm 261, 279-280.

6. Briaud I, Rouault C, Bailbe D, et al. (2000) Glucose induced insulin mRNA accumulation is impaired in islets from neonatal streptozotocin-treated rats. Horm Metab Res 32, 103-106.

7. Luo J, Yperselle MV, Rizkalla SW, et al. (2000) Chronic consumption of short-chain fructo-oligosaccharides does not affect basal hepatic glucose production or insulin resistance in type 2 diabetics. J Nutr 130, 1572-1577.

8. Trowell HC (1972) Ischemic heart disease and dietary fiber. Am J Clin Nutr 25, 926-932.

9. Bingham SA, Williams DRR \& Cummings JH (1985) Dietary fiber consumption in Britain: new stimates and their relation to large bowel cancer mortality. Br J Cancer 5, 399-402.

10. Gibson GR \& Roberfroid MB (1995) Dietary modulation of the human colonic microbiota: introducing the concept of prebiotics. J Nutr 125, 1401-1412.

11. Van Loo JP, Cousement P, De Leenheer L, et al. (1995) On the presence of inulin and oligofructose as natural ingredients in the western diet. Crit Rev Food Sci Nutr 35, 525-552.

12. Vazquez MJ, Alonso JL, Dominguez H, et al. (2000) Xylooligosaccharides: manufacture and applications. Trends Food Sci Technol 11, 387-393.

13. Campbell JM, Fahey GC \& Wolf BW (1996) Selected indigestible Oligosaccharides affect large bowel mass, cecal and fecal short-chain fatty acids, $\mathrm{pH}$ and microflora in rats. J Nutr 27, $130-136$.

14. Hsu CK, Liao JW, Chung YC, et al. (2004) Xylooligosaccharides and fructooligosaccharides affect the intestinal microbiota and precancerous colonic lesion development in rats. $J$ Nutr 134, 1523-1528.

15. Yamashita K, Kawai K \& Itakura M (1984) Effects of fructooligosaccharides on blood glucose and serum lipids in diabetic subjects. Nutr Res 4, 961-966.

16. Imaizumi K, Nakatsu Y, Sato M, et al. (1991) Effect of xylooligosaccharides on blood glucose, serum and liver lipid and cecal short chain fatty acid on diabetic rats. Agric Biol Chem 55, 199-205.

17. Sangeetha PT, Ramesh MN \& Prapulla SG (2002) Influence of media components and reaction parameters on the production of fructosyl transferase and fructooligosaccharides. Sci Aliment 22, 277-287.

18. Aachary AA \& Prapulla SG (2008) Corn-induced endo-1, 4- $\beta$-D-xylanase of Aspergillus oryzae MTCC 5154: production and characterization of xylobiose from Glucuronoxylan. J Agric Food Chem 56, 3981-3988. 
19. Jeong KJ, Park IY, Kim MS, et al. (1998) High-level expression of an endoxylanase from gene Bacillus sp. in Bacillus subtilis DB104 for the production of xylobiose from xylan. Appl Microbiol Biotechnol 50, 113-118.

20. Huggett ASG \& Nixon DA (1957) Use of glucose oxidase, peroxidase and $O$-dianisidine in the determination of blood glucose and urinary glucose. Lancet $\mathbf{2 7 3}, 366-370$.

21. Bradford MM (1976) A rapid and sensitive method for the quantitation of microgram quantities of protein utilizing the principle of protein-dye binding. Anal Chem 72, 248-254.

22. Oser BL (1965) In Hawk's Physiological Chemistry, 14th ed., p.1040. New Delhi: McGraw Hill Publishing Co.

23. Oser BL (1965) In Hawk's Physiological Chemistry, 14th ed., p.1039. New Delhi: McGraw Hill Publishing Co.

24. Wyebenga DR, Di Giorgio J \& Pileggi VJ (1971) Manual and automated methods for urea nitrogen measurement in whole serum. Clin Chem 17, 891-895.

25. Monnier VM \& Cerami A (1981) Nonenzymatic browning in vivo: possible process for aging of long-lived proteins. Science 211, 491-493.

26. Rudel LL \& Morris MD (1973) Determination of cholesterol using $o$-phthalaldehyde. J Lipid Res 14, 363-366.

27. Takahara S, Hamilton BH, Nell JV, et al. (1960) Hypocatalasemia, a new genetic carrier state. J Clin Invest 29, 610-619.

28. Racker E (1955) Glutathione reductase from bakers' yeast and beef liver. J Biol Chem 217, 855-865.

29. Mabel MJ, Sangeetha PT, Platel K, et al. (2008) Physiochemical characterization of fructooligosaccharides and evaluation of their suitability as a potential sweetener for diabetes. Carbohydr Res 343, 56-66.

30. Okazaki M, Fujikawa S \& Matsumoto N (1990) Effect of xylooligosaccharides on the growth of bifidobacteria. Bifidobact Microflora 9, 77-86.

31. Okazaki M, Koda H, Izumi R, et al. (1991) In vitro digestibility and in vivo utilization of xylobiose. J Jpn Soc Nutr Food Sci 44, 41-44.
32. Kleessen B, Hartmann L \& Blaut M (2001) Oligofructose and long-chain inulin: influence on the gut microbial ecology of rats associated with a human faecal flora. Br J Nutr 86, 291-300.

33. Venters CS, Vorster HH \& Cummings JH (1990) Effects of dietary propionate on carbohydrate and lipid metabolism in healthy volunteers. Am J Gastroenterol 85, 549-553.

34. Laurent C, Simoneau C, Marks L, et al. (1995) Effect of acetate and propionate on fasting hepatic glucose production in humans. Eur J Clin Nutr 49, 484-491.

35. Sheu WH-H, Lee I-Te, Chen W, et al. (2008) Effects of xylooligosaccharides on type 2 diabetes mellitus. J Nutr Sci Vitaminol 54, 396-401.

36. van Bennekum AM, Nruyen DV, Schulthess G, et al. (2005) Mechanisms of cholesterol-lowering effect of dietary insoluble fibres: relationships with intestinal and hepatic cholesterol parameters. Br J Nutr 95, 331-337.

37. Williams CM (1999) Effects of inulin on lipid parameters in humans. J Nutr 129, Suppl. 1, S1471-S1473.

38. Kok N, Roberfroid M, Robert A, et al. (1996) Involvement of lipogenesis in the lower VLDL secretion induced by oilgofructose in rats. $\mathrm{Br} J$ Nutr 76, 881-890.

39. Beylot M (2005) Effects of inulin-type fructans on lipid metabolism in man and in animal models. Br J Nutr 93, Suppl. 1, S163-S168.

40. Younes H, Garleb K, Behr S, et al. (1995) Fermentable fibers or oligosaccharides reduce urinary nitrogen excretion by increasing urea disposal in the rat cecum. $J$ Nutr $\mathbf{1 2 5}$, 1010-1016.

41. Henri K, Mule C, Svend-Aage J, et al. (1990) Relationship between serum cholesterol and diabetic nephropathy. Lancet ii, $1300-1304$.

42. Baynes JW (1991) Role of oxidative stress in development of complications in diabetes. Diabetes 30, 405-412. 